\title{
Early-stage Effects of Residual Charges in a Metal Target on Emitted Electrons Induced by Femtosecond Laser-Metal Interactions
}

\author{
${ }^{1}$ Sha Tao and ${ }^{2 *}$ Benxin Wu \\ 1: Advanced Optowave Corp., Ronkonkoma, NY \\ 2*: corresponding author; Associate professor, School of Mechanical Engineering, Purdue University, 585 Purdue \\ Mall, West Lafayette, IN 47907, email: wu65@ purdue.edu; Adjunct associate professor, Department of Mechanical, \\ Materials, and Aerospace Engineering, Illinois Institute of Technology, Chicago, IL 60616, email: bwu11@iit.edu.
}

\begin{abstract}
Electron emissions from a metal target surface may be induced due to the irradiation of the target by a femtosecond (fs) laser pulse. The emitted electrons will leave behind residual charges (which are positive) in the metal target near its surface. The residual charges may affect the evolution of the emitted electrons, which is called the "residual charge effect". An intuitive belief could be that the residual charge effect is insignificant, because the huge number of free electrons in the interior region of the metal may quickly neutralize the residual charges. In this paper, the early-stage (at a time scale of less than $\sim 1$ picosecond) residual charge effect has been investigated. The study shows that contrary to the above intuitive belief, the early-stage residual charge effect is very significant under the studied conditions, which has greatly slowed down the expansion of emitted electrons and enhanced their recombination back into the surface of the target. The study implies that to accurately study the early-stage fs laser-induced electron emission and other closely related processes, the residual charge effect should not be neglected.
\end{abstract}

Keywords: laser-induced electron emission; residual charge effect

\section{Introduction}

Femtosecond lasers are special energy sources that can deliver energies to a solid target in an ultra short amount of time, and have many applications in manufacturing, materials processing, biomedical, and other areas [1-6]. Numerous previous investigations have been performed on femtosecond (fs) laser-material interaction, including the electron emission process (and related processes) from a target surface induced by the irradiation of the surface by a fs laser pulse [7-16]. It has been found that the emitted electrons may feel the repulsive forces generated by the electrons emitted earlier, and hence their evolution may be affected, due to the so-called "space charge effect" [15-17]. Previous studies show that the space charge effect plays 
a very critical role in the electron emission induced by fs laser pulse-metal interactions [15-17].

The electron emission process may transiently break the electric neutrality in the metal target near-surface region, and leave positive residual charges inside the metal target near its surface. The residual charges in the metal target may also influence the electron emission and evolution process, which will be called the "residual charge effect" in this paper.

However, although research work has been carried out on the space charge effect during fs laser-produced electron emission from a metal target (for example, [15-17]), previous investigations about how significant the residual charge effect is during the early stage have been limited ("early stage" here means $t<\sim 1$ picosecond (ps), where $t=0$ is defined as the time when the fs laser-metal interaction starts). An intuitive belief could be that because a metal target has a huge number of free electrons with good mobilities, the surface residual charges may be quickly neutralized or re-distributed, making their effect on the emitted electrons insignificant. It will improve the basic understanding of fs laser-metal interactions to test this belief and reveal whether or not the early-stage "residual charge effect" is significant.

In this paper, research work will be performed to reveal the significance of the earlystage "residual charge effect" for fs laser-induced electron emission and evolution from a metal target. During the early stage, most of the emitted electrons may be in a close vicinity to the target surface (less than $\sim 1 \mu \mathrm{m}$ under the investigated conditions in this paper as shown later), and hence direct experimental measurements will require extremely high temporal and spatial resolutions, which is very challenging to achieve (even through the impressive time-resolved measurement technique using an ultrashort pulsed electron beam reported in [10-11], where the electron beam size is around $75 \mu \mathrm{m})$. Therefore, in this paper a physics-based model for the process will be employed as the investigation tool due to the measurement challenges mentioned 
above. The model calculations with and without considering the "residual charge effect" will be compared, which can reveal the significance of the effect during the early stage $(\mathrm{t}<1 \mathrm{ps})$. It will also be demonstrated later that the model prediction shows a reasonable consistency with a measurement result taken from the literature [15] on the number of emitted electrons due to fs laser pulse interaction with a metal.

\section{Model}

The modeling approach is similar to that in the authors' previous paper [18], where more details can be found. In this paper, only a brief introduction will be given due to the space limit. Please note that the authors' previous work in [18] is mainly to study the emitted electrons' effect on the electric field inside a metal target to provide a possible explanation of Coulomb explosion during fs laser-metal interaction, which is obviously very different from the focus of this paper.

In the model setup, it is assumed that a metal target is located in vacuum, and the metalvacuum interface is at $\mathrm{z}=0$, while the vacuum is in the domain of $\mathrm{z}>0$ and the metal is in the domain of $\mathrm{z}<0$. A fs laser pulse propagates in the $-\mathrm{z}$ direction and begins the interaction with the metal target at $t=0$. In the model, the changes of the electron and lattice temperatures inside the metal target are described through the two-temperature heat transfer equations, and the number density change of electrons in the metal target is described through the electron continuity equation $[7-9,15,19-23]$ :

$$
\begin{aligned}
& C_{e} \frac{\partial T_{e}}{\partial t}+C_{e} \frac{1}{e n_{e}}\left(-e n_{e} \mu_{e} E-e D \frac{\partial n_{e}}{\partial z}\right) \frac{\partial T_{e}}{\partial z}=\frac{\partial}{\partial z}\left(k_{e} \frac{\partial T_{e}}{\partial z}\right)-G\left(T_{e}-T_{l}\right)+S(z, t) \\
& C_{l} \frac{\partial T_{l}}{\partial t}=\frac{\partial}{\partial z}\left(k_{l} \frac{\partial T_{l}}{\partial z}\right)-G\left(T_{l}-T_{e}\right) \\
& \frac{\partial n_{e}}{\partial t}=\frac{\partial}{\partial z}\left(n_{e} \mu_{e} E+D \frac{\partial n_{e}}{\partial z}\right)
\end{aligned}
$$


where $C_{e}$ and $C_{l}$ denote the electron and the lattice heat capacity, respectively, $T_{e}$ and $T_{l}$ represent the electron and lattice temperature, respectively, $t$ is time, $S(z, t)$ denotes the source term due to the absorption of laser energy, $e$ represents the electronic charge magnitude, $n_{e}$ represents the number density of free electrons, $\mu_{e}$ denotes the electron mobility, $E$ is the electric field, $D$ denotes the diffusion coefficient, and is related to the electron mobility through $D=$ $k_{b} T_{e} \mu_{e} / e, k_{b}$ is the Boltzmann constant, $k_{e}$ is the thermal conductivity of electrons, $G$ is the electron-phonon coupling factor, $k_{l}$ is the lattice thermal conductivity, and in comparison with the electron thermal conduction, the lattice thermal conduction is often negligible in a metal target.

The electron emission at the target surface can be calculated as $[8-9,15-16,19,24-26]$ :

$$
J=A T_{e, S}^{2} \exp \left(-\frac{\varphi}{k_{b} T_{e, S}}\right)+\sum_{n=1}^{\infty} a_{n} A T_{e, S}^{2} I^{n}(1-R)^{n}\left(\frac{e}{h v}\right)^{n} F\left(\frac{n h v-\varphi}{k_{b} T_{e, S}}\right)
$$

where $\mathrm{J}$ is the total electron emission current density, and the first term on the right side of the equation represents the contribution due to thermionic emission, while the other terms on the right side represent the contribution due to photoemission, and $T_{e, S}$ represents the temperature of electrons at the target surface, $A$ and $\varphi$ denote the theoretical Richardson coefficient, and the work function respectively, $I$ and $\mathrm{R}$ are the laser beam intensity and target surface reflectivity, respectively (and hence $I(I-R)$ is the absorbed laser intensity), $h$ and $v$ are the Planck constant, and laser photon frequency, respectively (and hence $h v$ is the laser photon energy), $a_{n}$ represents a constant, and $F(x)$ denotes the Fowler function.

The finite difference method has been applied to solve the governing equations in the metal target [27]. The evolution of the electrons emitted into the vacuum domain of $z>0$ is modeled using the "particle-in-cell" (PIC) method [15-16, 28-29]. The velocity distributions of 
electron macro particles emitted at the target surface are determined based on the assumption that the electrons in the metal target follow the Fermi-Dirac statistics [30-31]. During the electron evolution in the vacuum, some electron macro particles may move back into the target surface, which has been considered in the model calculations. The electric field in both the metal target and the vacuum region is determined by solving the Poisson's equation based on the spatial distribution of electric charges $[8,32]$. Based on the electric field, the force exerted on each electron macro particle can be determined, using which the updated velocity and position of each electron macro particle after one numerical time step can be determined by solving the particle's equation of motion. The updated electron macro particles' positions will lead to an updated spatial distribution of the electric charge, based on which the updated electric field can be determined by solving the Poisson's equation. This process can be repeated for each numerical time step during the simulation.

Aluminum is chosen as the target material, and the major material properties and parameters are taken from the literature $[9,15,17,20,23,33-35]$ (see the authors' previous paper [18] for detailed values).

\section{Results and Discussions}

Figure 1 shows the model-predicted emitted electron number density distributions above the target surface in the vacuum domain at $\mathrm{t}=100 \mathrm{fs}$, which is due to the interaction of a 90 -fs laser pulse with an aluminum target. Based on the above introduction about the model, it can be seen that both the space charge effect and the residual charge effect have been considered in the model. As a comparison, Fig. 1 has also shown the results of the simulation that has neglected the residual charge effect and has only considered the space charge effect (where the residual 
charge effect is neglected by assuming that the electron number density in the target always remains at its initial value, and also by neglecting the second term on the left side of Eq.(1)). It can be seen from Fig. 1 that when both the space charge effect and the residual charge effect are considered, most of the emitted electrons are within around $\sim 50 \mathrm{~nm}$ above the target surface at $\mathrm{t}$ $=100 \mathrm{fs}$. The electron number density drops quickly with $\mathrm{z}$, and has a very high spatial gradient in $+\mathrm{z}$ direction. On the other hand, if only the space charge effect is considered and the residual charge effect is neglected, the emitted electrons expand much faster. At $t=100 \mathrm{fs}$, the emitted electron front of $\mathrm{n}_{\mathrm{e}}=10^{20} / \mathrm{m}^{3}$ has expanded beyond $\mathrm{z}=\sim 550 \mathrm{~nm}$. Due to the fast expansion, the spatial distribution of the electron density becomes relatively more uniform.

The results in Fig.1 are significantly different from the intuitive belief that because a metal target has a huge number of free electrons with good mobilities, the surface residual charges will be quickly neutralized, making their effect on the emitted electrons insignificant. Instead, Fig. 1 clearly shows that the positive residual charges in the metal target near its surface have significantly slowed down the expansion of the emitted electrons during the early stage shown in the figure, which has led to a very thin layer of emitted electrons in the vacuum domain near the target surface with a large spatial gradient of the electron density.

Figure 2a shows the model-predicted front locations (defined as the front locations where $\mathrm{n}_{\mathrm{e}}=10^{20} / \mathrm{m}^{3}$ and $10^{18} / \mathrm{m}^{3}$, respectively) of emitted electrons, where both the space charge effect and the residual charge effect have been considered. Figure $2 b$ shows the front locations when only the space charge effect is considered and the residual charge effect is neglected. It can be seen that when the residual charge effect is considered, the front of emitted electrons only reaches $\mathrm{z}=\sim 520 \mathrm{~nm}\left(\right.$ for $\left.\mathrm{n}_{\mathrm{e}}=10^{20} / \mathrm{m}^{3}\right)$ and $\mathrm{z}=\sim 760 \mathrm{~nm}\left(\right.$ for $\left.\mathrm{n}_{\mathrm{e}}=10^{18} / \mathrm{m}^{3}\right)$ at $\mathrm{t}=1 \mathrm{ps}$. However, if the residual charge effect is neglected, Fig. $2 \mathrm{~b}$ shows that the front approaches $\mathrm{z}=\sim 34 \mu \mathrm{m}$ at $\mathrm{t}$ 
$=1 \mathrm{ps}$, where the front propagation distance is much larger than that when the residual charge effect is considered. Figure 2 shows again that the residual charge effect has significantly slowed down the expansion of the emitted electrons during the early stage shown in the figure.

The results in Figs. 1 and 2 show that during the early stage of $t<\sim 1$ ps, most of the emitted electrons are still positioned closely right above the surface of the target in a layer that is very narrow $(<\sim 1 \mu \mathrm{m}$ for the studied case). This implies an extremely high difficulty for direct experimental measurements, which is why a physics-based model is employed in this study.

Figure 3 shows the total number of emitted electrons versus time predicted by the model calculations. The total number will first quickly increase with time, and then decrease with time due to the electrons returning to the surface of the target. Figure 3 shows that the residual charge effect has played a very critical role in the recombination of the emitted electrons back into the surface of the target. The overall net rate of recombination, when considering the residual charge effect, is much higher than that without considering the effect. By $t=1 \mathrm{ps}$, due to the recombination the total number of emitted electrons has dropped to $\sim 8.5 \times 10^{6}$ when the residual charge effect is considered, while the total number still maintains at a much higher value of $\sim 7.7 \times 10^{8}$ when the effect is neglected. Hence, Figure 3 shows that the residual charge effect has significantly enhanced the recombination of emitted electrons back into the target surface during the early stage shown in the figure.

As discussed in the authors' previous paper [18], the employed model has its obvious limit due to some material properties or related parameters' accuracy uncertainties and/or limited availabilities, the model's continuum (target portion) and 1D (one-dimensional) nature, and the limited numerical accuracy and resolution due to the restriction of the associated highcomputational cost, etc. However, it is expected that the model should be sufficient to achieve 
the major goal of this work, which is to qualitatively reveal the significance of the residual charge effect during the early stage $(\mathrm{t}<1 \mathrm{ps})$, instead of performing quantitatively fully accurate model calculations.

Finally, Figure 4 shows the comparison between the model-predicted temporal variation of the total number of emitted electrons (where both the space charge and residual charge effects have been considered) and the final total electron yield that is measured in and taken from [15]. The figure shows that the model-predicted total number of emitted electrons first increases and then decreases with time due to the electron recombination. By $\mathrm{t}=\sim 20 \mathrm{ps}$, the net electron recombination rate is significantly lower than its peak value during the earlier stage, and the decreasing of the total electron number becomes much slower, and at $t=\sim 20$ ps the modelpredicted total number of emitted electrons is reasonably close to the final total electron yield that is measured in and taken from [15]. Due to the high computational cost in the PIC part and the employed model's 1D nature, the simulation using the model can not be carried out for a very long period. However, Figure 4 does show a reasonable consistency between the prediction by the model and the given measurement on the emitted electron number under the given condition. When the model calculates the transient total number of emitted electrons shown in Figs.3 and 4, the assumed laser spot area is the same as that in [15].

\section{Conclusion}

In conclusion, under the investigated conditions it has been found that the positive residual charges in the metal target near its surface significantly affect the evolution of emitted electrons produced by a fs laser pulse interactions with the target during the early stage $(\mathrm{t}<1 \mathrm{ps})$. Due to the residual charge effect, the expansion of the emitted electrons has been significantly 
slowed down, and the recombination of the emitted electrons back into the surface of the target has been greatly enhanced. The result implies that to accurately study the early-stage electron emission and other closely related processes or phenomena due to fs laser pulse-metal interactions, the residual charge effect should not be neglected.

\section{Acknowledgement}

This material is based upon work supported by the National Science Foundation under Grant No. CBET-0853528. 


\section{List of References}

1. N.B. Dahotre and S.P. Harimkar, Laser Fabrication and Machining of Materials (Springer Science \& Business Media, 2008).

2. L. Jiang, D. Ying, X. Li, and Y. Lu, Opt. Lett. 37, 3648-3650 (2012)

3. H.Huang and Z. Guo, J. Phys. D: Appl. Phys. 42, 165204 (2009).

4. X. Wang and X. Xu, Journal of Thermal Stresses 25, 457-473 (2002).

5. W. Zhang and S. Chen, MRS Bulletin 36, 1028-1033 (2011).

6. Q. Bian, X. Yu, B. Zhao, Z. Chang, and S. Lei, Optics \& Laser Technology 45, 395-401 (2013).

7. R. Stoian, A. Rosenfeld, D. Ashkenasi, I. V. Hertel, N. M. Bulgakova, and E. E. B. Campbell, Phys. Rev. Lett. 88, 097603 (2002).

8. N. M. Bulgakova, R. Stoian, A. Rosenfeld, I. V. Hertel, and E. E. B. Campbell, Phys. Rev. B 69, 054102 (2004).

9. N. M. Bulgakova, R. Stoian, A. Rosenfeld, I. V. Hertel, W. Marine, and E. E. B. Campbell, Appl. Phys. A 81, 345-356 (2005).

10. J. Li, X. Wang, Z. Chen, J. Zhou, S. S. Mao, and J. Cao, Appl. Phys. Lett. 98, 011501 (2011).

11. J. Li, X. Wang, Z. Chen, R. Clinite, S.S. Mao, P. Zhu, Z. Sheng, J. Zhang and J. Cao, J. Appl. Phys. 107, 083305 (2010).

12. M. Hashida, S. Namba, K. Okamuro, S. Tokita, and S. Sakabe, Phys. Rev. B 81, 115442 (2010).

13. H. Dachraoui and W. Husinsky, Appl. Phys. Lett. 89, 104102 (2006).

14. H. Dachraoui, W. Husinsky, and G. Betz, Appl. Phys. A 83, 333-336 (2006).

15. D. M. Riffe, X. Y. Wang, M. C. Downer, D. L. Fisher, T. Tajima, J. L. Erskine, and R. M. More, J. Opt. Soc. Am. B 10, 1424-1435 (1993).

16. W. Wendelen, D. Autrique, and A. Bogaerts, Appl. Phys. Lett. 96, 051121(2010).

17. W. Wendelen, B.Y. Mueller, D. Autrique, B. Rethfeld, and A. Bogaerts, J. Appl. Phys. 111, 113110 (2012).

18. S. Tao, and B. Wu, Applied Surface Science, 298, 90-94 (2014).

19. Z. Chen and S. S. Mao, Appl. Phys. Lett. 93, 051506 (2008).

20. Z. Lin, L. V. Zhigilei, and V. Celli, Phys. Rev. B 77, 075133 (2008).

21. S. I. Anisimov and B. Rethfeld, SPIE Proceedings 3093, Nonresonant Laser-Matter Interaction (NLMI-9), 192-203 (April 4, 1997).

22. S. Nolte, C. Momma, H. Jacobs, A. Tünnermann, B. N. Chichkov, B. Wellegehausen, and H. Welling, J. Opt. Soc. Am. B 14, 2716-2722 (1997).

23. G. Tas and H. J. Maris, Phys. Rev. B 49, 15046-15054 (1994).

24. T. Balasubramani and S. H. Jeong, J. Phys.: Conference Series 59, 595-599 (2007).

25. J. H. Bechtel, W. Lee Smith, and N. Bloembergen, Phys. Rev. B 15, 4557-4563 (1977).

26. P. Martin, R. Trainham, P. Agostini, and G. Petite, Phys. Rev. B 45, 69-77 (1992). 
27. F. P. Incropera, D. P. DeWitt, T.L. Bergman, and A.S. Lavine, Fundamentals of Heat and Mass Transfer (John Wiley \& Sons, Inc., Hoboken, New Jersey, 2007).

28. W. Hu, Y. C. Shin, and G. King, Phys. Plasmas 18, 093302 (2011).

29. C. K. Birdsall and A. B. Langdon, Plasma Physics via Computer Simulation (Taylor \& Francis, New York, 2005).

30. R.H. Fowler, Phys. Rev. 38, 45-56 (1931).

31. L.A. Dubridge, Phys. Rev. 43, 727-741 (1933).

32. P. Hammond, Electromagnetism for Engineers: An Introductory Course (Pergamon Press, Oxford, 1978).

33. N. W. Ashcroft and N. D. Mermin, Solid State Physics (Harcourt, Inc., Orlando, Florida, 1976).

34. R.A. Serway, Principles of Physics (Fort Worth: Saunders College Pub., 1998).

35. J. P. Colombier, P. Combis, F. Bonneau, R. Le Harzic, and E. Audouard, Phys. Rev. B $71,165406(2005)$. 


\section{List of Figure Captions}

Figure 1 . The density spatial distributions in the vacuum domain for emitted electrons at $\mathrm{t}=100$ fs, predicted by the model calculations with and without considering the residual charge effect (laser pulse duration: $90 \mathrm{fs}$, fluence: $17.7 \mathrm{~mJ} / \mathrm{cm}^{2}$; the aluminum target surface is located at $\mathrm{z}=0$ )

Figure 2. The emitted electron front locations (defined as the front locations where $n_{\mathrm{e}}=10^{20} / \mathrm{m}^{3}$ and $10^{18} / \mathrm{m}^{3}$, respectively) versus time predicted by the model calculations: (a) considering both the space charge effect and the residual charge effect, and (b) only considering the space charge effect (the simulated conditions are the same as Fig. 1; for (b) the front locations for $n_{\mathrm{e}}=10^{20} / \mathrm{m}^{3}$ and $10^{18} / \mathrm{m}^{3}$ are very close to each other at $\mathrm{t} \geq 300 \mathrm{fs}$ )

Figure 3. The total number of emitted electrons versus time predicted by the model calculations with and without considering the residual charge effect (the simulated conditions are the same as Fig.1)

Figure 4. The temporal variation of the total number of emitted electrons due to fs laser pulse irradiation of an aluminum target located in vacuum predicted by the model calculation in this paper, in comparison with the total final electron yield that is measured in and taken from [15] (The assumed conditions in the model are the same as Figs.1 to 3. See [15] for more details on the experimental conditions. In [15], the measured electron yield is presented in a logarithmic scale plot, and small uncertainties may exist when the data in the plot is converted to numerical values of electron yield vs laser pulse fluence). 


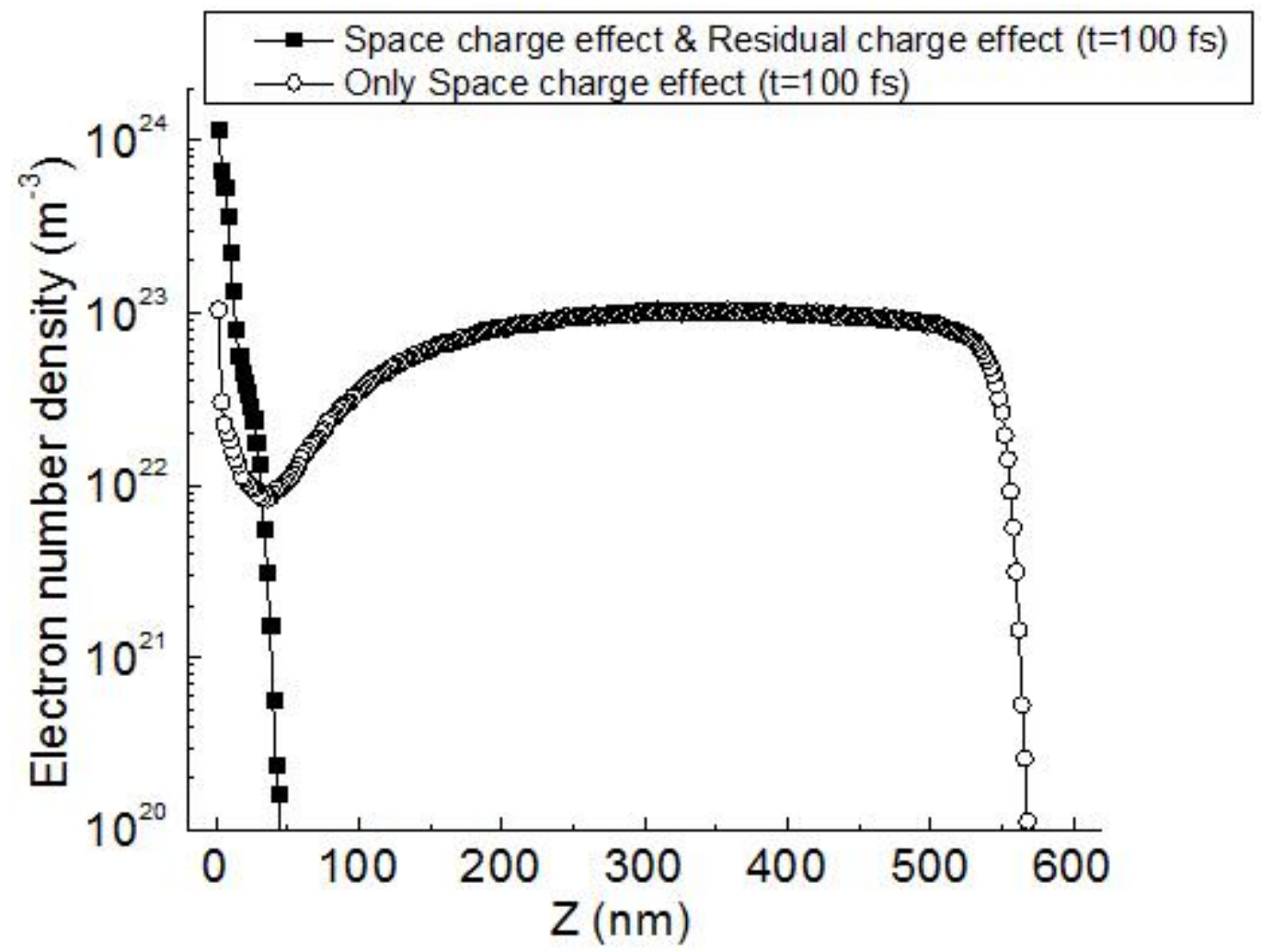

Figure 1 . The density spatial distributions in the vacuum domain for emitted electrons at $t=100 \mathrm{fs}$, predicted by the model calculations with and without considering the residual charge effect (laser pulse duration: 90 fs, fluence: $17.7 \mathrm{~mJ} / \mathrm{cm}^{2}$; the aluminum target surface is located at $\mathrm{z}=0$ ) 


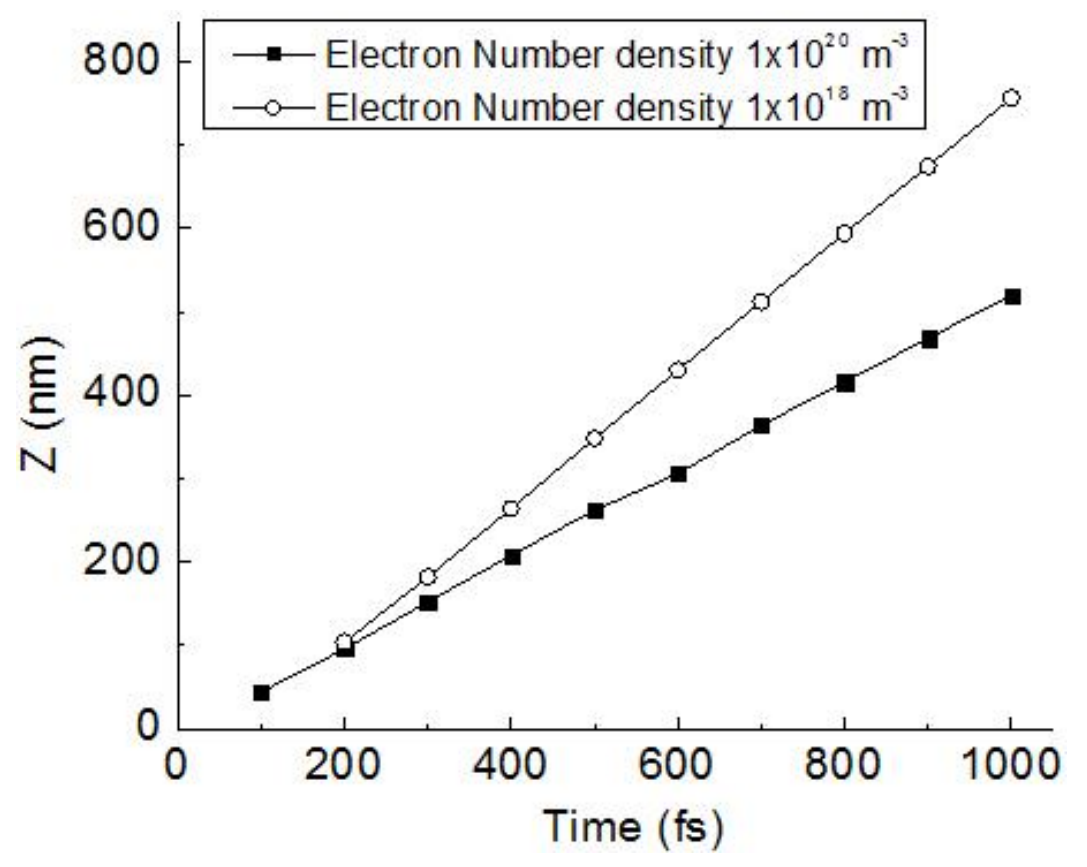

(a)

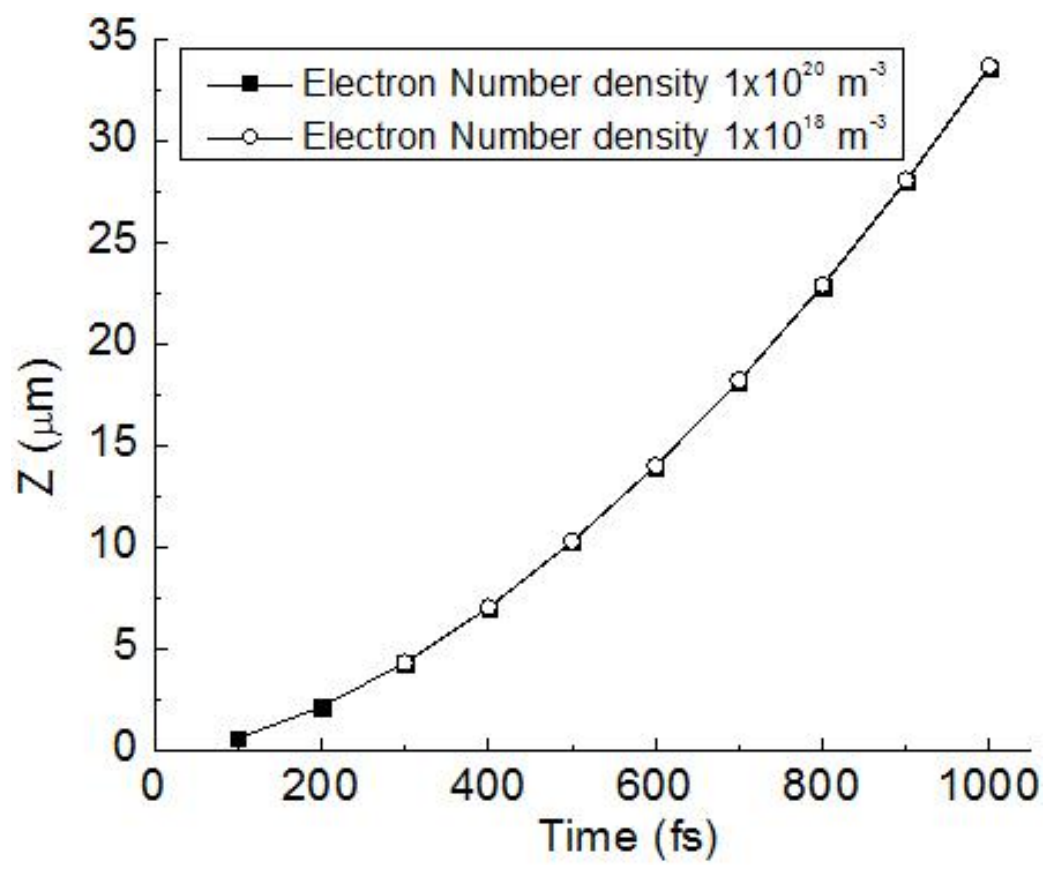

(b)

Figure 2. The emitted electron front locations (defined as the front locations where $\mathrm{n}_{\mathrm{e}}=10^{20} / \mathrm{m}^{3}$ and $10^{18}$ $/ \mathrm{m}^{3}$, respectively) versus time predicted by the model calculations: (a) considering both the space charge effect and the residual charge effect, and (b) only considering the space charge effect (the simulated conditions are the same as Fig. 1; for (b) the front locations for $n_{e}=10^{20} / \mathrm{m}^{3}$ and $10^{18} / \mathrm{m}^{3}$ are very close to each other at $t \geq 300 \mathrm{fs}$ ) 


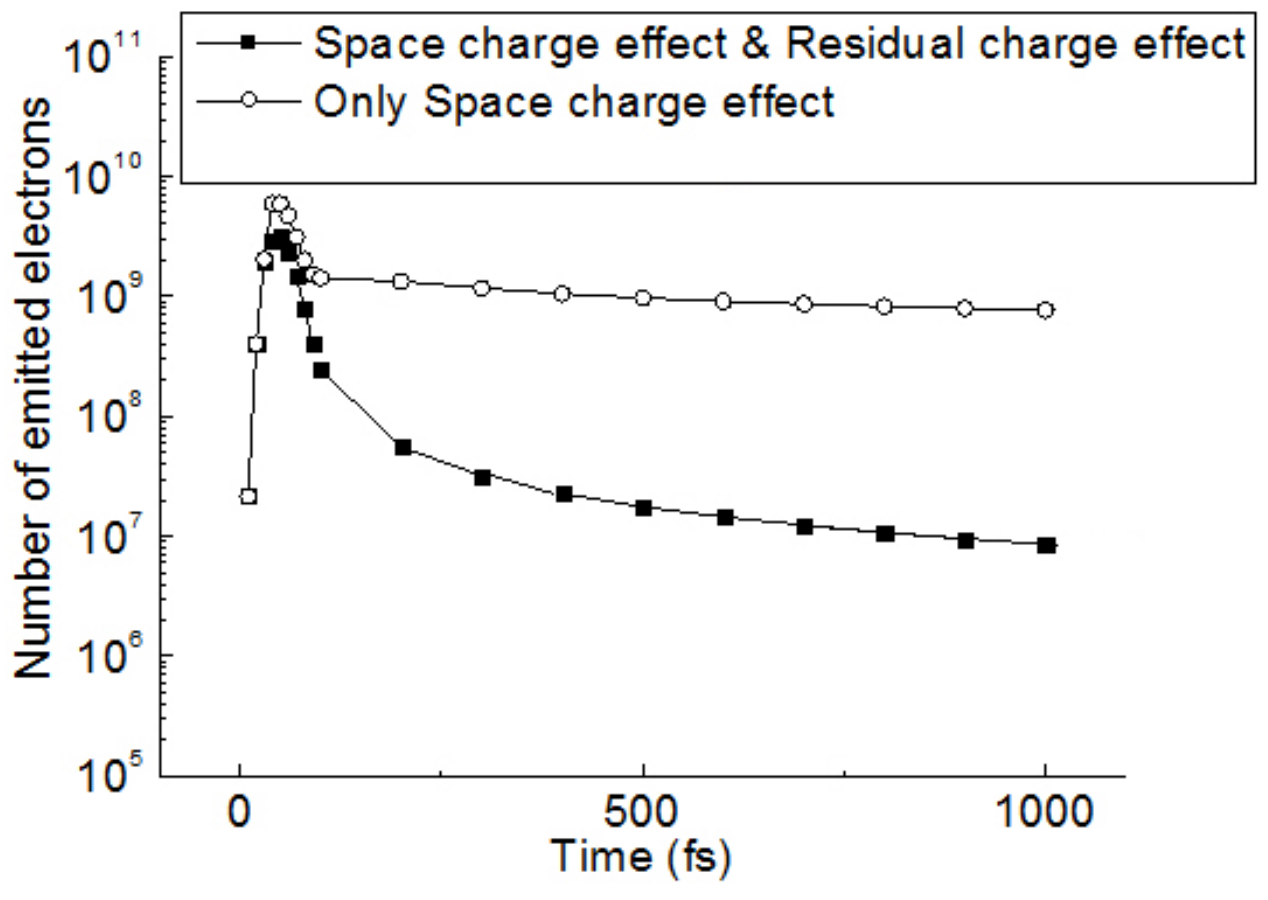

Figure 3. The total number of emitted electrons versus time predicted by the model calculations with and without considering the residual charge effect (the simulated conditions are the same as Fig.1) 


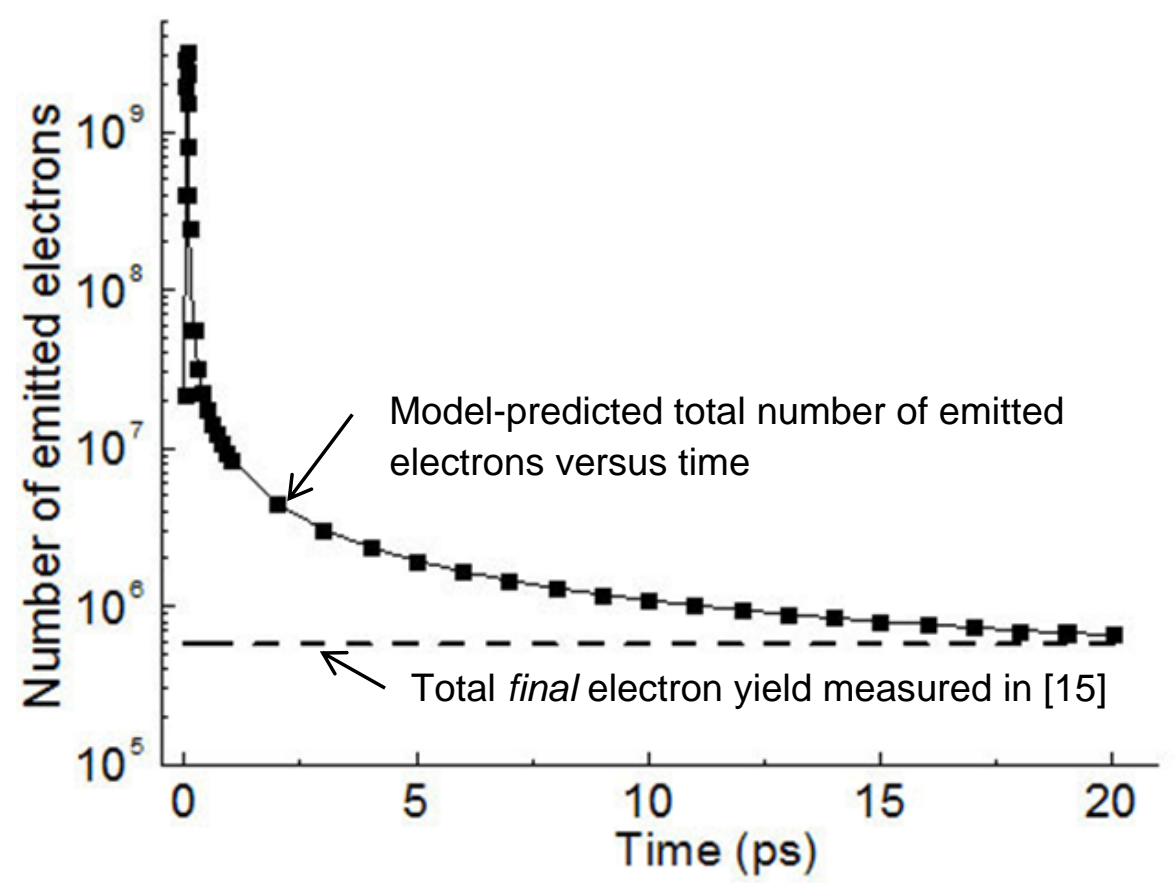

Figure 4. The temporal variation of the total number of emitted electrons due to fs laser pulse irradiation of an aluminum target located in vacuum predicted by the model calculation in this paper, in comparison with the total final electron yield that is measured in and taken from [15] (The assumed conditions in the model are the same as Figs.1 to 3. See [15] for more details on the experimental conditions. In [15], the measured electron yield is presented in a logarithmic scale plot, and small uncertainties may exist when the data in the plot is converted to numerical values of electron yield vs laser pulse fluence). 